\title{
edoc
}

Institutional Repository of the University of Basel

University Library

Schoenbeinstrasse 18-20

CH-4056 Basel, Switzerland

http://edoc.unibas.ch/

Year: 2010

\section{Engineering human cell-based, functionally integrated osteochondral grafts by biological bonding of engineered cartilage tissues to bony scaffolds}

Scotti, Celeste and Wirz, Dieter and Wolf, Francine and Schaefer, Dirk J. and Bürgin, Vivienne and Daniels, Alma U. and Valderrabano, Victor and Candrian, Christian and Jakob, Marcel and Martin, Ivan and Barbero, Andrea.

Posted at edoc, University of Basel

Official URL: http://edoc.unibas.ch/dok/A6003827

Originally published as:

Scotti, Celeste and Wirz, Dieter and Wolf, Francine and Schaefer, Dirk J. and Bürgin, Vivienne and Daniels, Alma U. and Valderrabano, Victor and Candrian, Christian and Jakob, Marcel and Martin, Ivan and Barbero, Andrea. (2010) Engineering human cell-based, functionally integrated osteochondral grafts by biological bonding of engineered cartilage tissues to bony scaffolds. Biomaterials, Vol. 31, no. 8. S. 2252-2259. 


\title{
ENGINEERING HUMAN CELL-BASED, FUNCTIONALLY INTEGRATED \\ OSTEOCHONDRAL GRAFTS BY BIOLOGICAL BONDING OF ENGINEERED CARTILAGE TISSUES TO BONY SCAFFOLDS
}

\author{
Scotti C. ${ }^{1,2}$; Wirz D. ${ }^{3}$; Wolf F. ${ }^{1}$; Schaefer D.J. ${ }^{1,4}$; Bürgin V. ${ }^{3}$; Daniels A.U. ${ }^{3}$; \\ Valderrabano V. ${ }^{1,5}$; Candrian C. ${ }^{1,6}$; Jakob M. ${ }^{1,5}$; Martin I. ${ }^{* 1}$ and Barbero A. ${ }^{1}$
}

${ }^{1}$ Departments of Surgery and of Biomedicine, University Hospital Basel, Switzerland

${ }^{2}$ Residency Program in Orthopaedics and Traumatology, Università degli Studi di Milano, Italy

${ }^{3}$ Laboratory of Biomechanics \& Biocalorimetry, Faculty of Medicine, University of Basel, Switzerland

${ }^{4}$ Department of Plastic, Reconstructive and Aesthetic Surgery, University Hospital Basel, Switzerland

${ }^{5}$ Department of Ortopaedic Surgery, University Hospital Basel, Switzerland

${ }^{6}$ Department of Surgery, Ospedale Regionale di Lugano, Switzerland

*Corresponding Author: Ivan Martin

ICFS, University Hospital Basel

Hebelstrasse 20, ZLF, Room 405

4031 Basel, Switzerland

tel: + 4161265 2384; fax: + 41612653990

e-mail: $\underline{\text { imartin@uhbs.ch }}$

Running title: Engineering of human cell-based osteochondral graft 


\section{INTRODUCTION}

Articular cartilage is a highly specialized tissue, characterized by unique biomechanical properties and, on the other hand, a poor regenerative potential [1,2]. As a consequence, when a traumatic lesion or a non-traumatic disease such as osteochondritis dissecans occurs, the defect is filled with a fibrous tissue which is not capable to withstand the high compressive and shear forces acting in the joint, often leading to the development of early osteoarthritis [1]. An established surgical technique for the repair of osteochondral (OC) defects (i.e., mosaicplasty) involves transplantation of autologous OC plugs consisting of an articular cartilage layer and the underlying subchondral bone [3,4]. Despite the promising results reported, the use of autologous OC grafts suffers from several limitations, namely (i) amount of material available, (ii) donor site morbidity, and (iii) difficult graft/injured site matching. In vitro fabrication of OC composites of predefined size and shape starting from appropriate scaffolds, possibly combined with autologous cells, has the potential to overcome these limits [5].

To date, many tissue engineering approaches have been reported in literature to engineer OC grafts, both scaffold-free [6-8] or scaffold-based [9-12] for the chondral layer and cellfree [12-14] or cell-seeded [10,11,15] for the bony layer. Also biphasic OC scaffolds have been proposed [15-17].

The clinical translation of such strategies however is limited by the fact that (i) animal cells are often used instead of human cells [6,9]; (ii) the size of the graft is too small to be clinically relevant [6]; and (iii) the biomaterials used are not currently implanted in human patients. Moreover, the functionality of an engineered OC graft should be supported by (i) an efficient integration between the cartilage and bony layers, in order to withstand the shear forces acting in the joint, and at the same time (ii) a satisfactory maturation of the chondral layer, in order to allow partial loading and thus reduce post-operative immobilization. A chemical or physical bonding of the biomaterials would not necessarily guarantee a long 
lasting integration of the chondral and bony layers, especially if the degradation of the materials is not accompanied by an efficient neo-formation of extracellular matrix at the interface. Instead, a "biological bonding” between the two layers, namely their integration through the extracellular matrix synthesized by cultured cells, would provide an actively and durably interconnected interface even following the resorption/degradation of the biomaterials.

In this study, we aimed at developing and validating a method to engineer OC constructs based on the principle of biological bonding, and using clinically relevant cells and biomaterials. In particular, our strategy consisted in coupling a human adult chondrocytes (HAC)-seeded collagen type I/III matrix (Chondro-Gide ${ }^{\circledR}$, Geistlich Pharma AG, Switzerland) with a cell-free devitalized bovine trabecular bone cylinder (Tutobone ${ }^{\circledR}$, Tutogen Medical GmbH, Neuenkirchen am Brand, Germany) using fibrin glue (Tisseel ${ }^{\circledR}$, Baxter Healthcare, Newbury, UK). Chondro-Gide ${ }^{\circledR}$, Tutobone ${ }^{\circledR}$ and Tisseel ${ }^{\circledR}$ are extensively used biomaterials in clinical practice for cartilage repair, bone reconstruction and wound healing, respectively.

We opted for a cell-free bony layer since osteoprogenitor cells, abundantly present in the bleeding subchondral bone during implantation, are expected to colonize the scaffold and efficiently promote new bone formation $[12,16,18]$. We then developed a mechanical test and used it to address whether pre-culture time of chondrocyte-seeded matrices before combination with bony layers modulates the extent of integration between the two layers of resulting OC constructs. Finally, we investigated the quality of the chondral layer and whether it is affected by the presence of devitalized bone in culture, as previously described [9]. 


\section{MATERIALS AND METHODS}

\section{Cartilage biopsies, articular chondrocytes isolation and expansion}

Cartilage tissues without signs of osteoarthritis were collected from the femoral condyles of 5 cadavers (3 males and 2 females; mean age: 57 years; range 31-81 years) without macroscopic sign of arthritis, following informed consent by relatives and in accordance with the Local Ethical Committee. Cartilage tissues were weighed, minced into small pieces and digested with $0.15 \%$ type II collagenase (10 mL solution/g tissue) for 22 hours. The isolated human articular chondrocytes (HAC) were expanded for two passages with Dulbecco's Eagle’s Medium (DMEM) containing $4.5 \mathrm{mg} / \mathrm{mL}$ D-glucose, $0.1 \mathrm{mM}$ nonessential amino acids, $1 \mathrm{mM}$ sodium pyruvate, $100 \mathrm{mM}$ HEPES buffer, $100 \mathrm{U} / \mathrm{mL}$ penicillin, $100 \mu \mathrm{g} / \mathrm{mL}$ streptomycin and $0.29 \mathrm{mg} / \mathrm{mL}$ L-glutamate and supplemented with $10 \%$ of foetal bovine serum (complete medium-CM), 1 ng/mL of Transforming Growth Factor $\beta 1$ (TGF $\beta-1$ ), and 5 ng/mL of Fibroblast Growth Factor 2 (FGF-2) (all from R\&D Systems), as previously described [19].

\section{Generation of osteochondral samples}

Collagen type I/III matrices (Chondro-Gide ${ }^{\circledR}$, Geistlich Pharma AG), cored out in the shape of discs (11 mm diameter), were used as scaffolds for the chondral layer. The bony layer consisted of devitalized bovine cancellous bone cylinders (11 mm diameter, $10 \mathrm{~mm}$ thickness) (Tutobone $^{\circledR}$, Tutogen Medical GmbH). In Group 1, expanded HAC were detached from culture flasks and suspended in a $40 \mathrm{mg} / \mathrm{ml}$ fibrinogen solution (Tisseel ${ }^{\circledR}$, Baxter). Fibrinogen-embedded HAC were then loaded statically on the matrices, which were previously placed at the bottom of the wells of a custom-shaped silicon mould, at a density of $12 \times 10^{6}$ cells / $50 \mu \mathrm{l}$ fibrinogen solution (Fig. 1A). The bony cylinders, pre-wetted in $8 \mathrm{U} / \mathrm{ml}$ thrombin solution (Tisseel ${ }^{\circledR}$, Baxter), were placed on the cell-seeded matrix using a gentle finger pressure. After 20 minutes of polymerization, the combined constructs were extracted from the mould, transferred to culture flasks and cultured in CM supplemented with $0.1 \mathrm{mM}$ 
ascorbic acid (Sigma), $10 \mu \mathrm{g} / \mathrm{mL}$ Insulin (Actrapid ${ }^{\circledR}$, Novo Nordisk) and $10 \mathrm{ng} / \mathrm{mL}$ Transforming Growth Factor- $\beta 3$ (R\&D Systems) (Chondrogenic medium) in a humidified $37^{\circ} \mathrm{C} / 5 \% \mathrm{CO}_{2}$ incubator. For Group 2 and Group 3 constructs, matrices were statically loaded with cells and combined with the bony scaffolds after being pre-cultured for 3 or 14 days in Chondrogenic medium, using fibrin glue as described above, but without cells in the fibrinogen solution. Constructs were cultured up to 5 weeks, with media changes twice a week.

\section{Development of a peel-off test for mechanical integration}

A peel-off test was developed to allow for precise measurement of the integration force between biphasic tissue-engineered constructs. The peel-off test was based on the American Society for Testing and Materials (ASTM) International (West Conshohocken, Pennsylvania, USA) test standard D 3330, designed for evaluating peel-off strength of thin adhesive films [20]. In contrast to a T peel test described in the literature [21,22], allowing to peel apart two layers of articular cartilage, the peel-off test developed here was designed to detach a cartilaginous layer from the underlying bone-replacement material. The peel-off test was developed and performed on a small-scale mechanical testing machine (MTS Synergie 100, MTS Systems, Inc. Eden Prairie, Minnesota, USA) equipped with a 2 N load cell. The bottom surface of the bony layer of an OC construct was attached to a glass plate with cyanoacrylate glue and the plate was secured to the steel platen of the testing machine. The edge of the chondral layer was grasped with a small surgical clamp attached to the crosshead of the testing machine (Fig. 2A). The crosshead was raised at $1 \mathrm{~mm} / \mathrm{s}$, and force and displacement were recorded until full detachment of the chondral layer occurred. Parameters assessed were maximum peel off force, and total peel energy, normalized to the interfacial areas of the samples.

\section{Histological and immunohistochemical analyses}


Generated constructs were cut in half top-to-bottom, rinsed with PBS, fixed in 4\% formalin and embedded in methylmetacrylate for histological analysis (Safranin-O for sulfated glycosaminoglycans - GAG -) or in paraffin for immunohistochemical examination. Sections of $7 \mu \mathrm{m}$ thickness were processed for immunohistochemistry using antibodies anti type II collagen (CII) (II-II6B3, Hybridoma Bank, University of Iowa, USA) or type I collagen (CI) (Quartett Immunodiagnostika und Biotechnologie GmbH, Berlin, Germany), as previously described [23].

\section{Biochemical Analyses}

Chondral and bony layers, delaminated during peel-off tests of OC constructs, as well as chondral-only controls, were digested for 15 hours at $56^{\circ} \mathrm{C}$ with protease $\mathrm{K}(1 \mathrm{~mL}$ for the chondral layer and $2 \mathrm{~mL}$ for the bony layer of $1 \mathrm{mg} / \mathrm{mL}$ protease $\mathrm{K}$ in $50 \mathrm{mM}$ Tris with $1 \mathrm{mM}$ EDTA, $1 \mathrm{mM}$ iodoacetamide and $10 \mu \mathrm{g} / \mathrm{mL}$ pepstatin-A, respectively). GAG amounts were measured spectrophotometrically after reaction with dimethylmethylene blue, with chondroitin sulfate as a standard. DNA was measured spectrofluorometrically using the CyQuant cell proliferation assay Kit (Molecular Probes, Eugene, OR), with calf thymus DNA as a standard.

\section{Real-Time quantitative Reverse Transcriptase Polymerase Chain Reaction (RT-PCR)}

Total RNA was extracted from chondral and bony layers delaminated during the peel-off tests, as well as from chondral control samples (Fig. 1), using Trizol (Life Technologies, Basel, Switzerland) and the standard single-step acid-phenol guanidinium method. Samples were first sonicated for 1 min while in Trizol. RNA was treated with DNAseI using the DNAfreeTM Kit (Ambion, USA) and quantified spectrofluorimetrically. cDNA was generated from $3 \mathrm{mg}$ of RNA by using $500 \mathrm{mg} / \mathrm{mL}$ random hexamers (Catalys AG, CH) and $1 \mathrm{~mL}$ of 50 $\mathrm{U} / \mathrm{mL}$ StratascriptTM reverse transcriptase (Stratagene, NL), in the presence of dNTPs. PCR reactions were performed and monitored using the ABI Prism 7700 Sequence Detection System (Perkin-Elmer/Applied Biosystems, Rotkreuz, Switzerland). Cycle temperatures and 
times as well as primers and probes used for the reference gene (GAPDH) and the genes of interest (collagen types I, II, X, and Bone sialoprotein - BSP -), were as previously described $[19,24]$. For each cDNA sample, the threshold cycle (Ct) value of GAPDH was subtracted from the Ct value of the target gene, to derive $\Delta \mathrm{Ct}$. The level of expression of the genes of interest was calculated as $2^{\Delta \mathrm{Ct}}$.

\section{Statistical analysis}

For each analysis, at least triplicate pellets for each condition and donor were assessed. Statistical evaluation was performed using SPSS software version 7.5 software (SPSS, Sigma Stat). Values are presented as mean \pm standard deviation (SD). Differences between groups were assessed by Mann-Whitney test and considered statistically significant when $\mathrm{P}$ values were lower than 0.05 .

\section{RESULTS}

\section{Validation of peel-off tests for mechanical integration}

Using cell-free OC constructs, we first tested two geometrical configurations, which based on the angle between the OC surface and the applied force are referred to as $90^{\circ}$ peel-off or $180^{\circ}$ peel-off (Fig. 2A). Both $90^{\circ}$ peel-off and $180^{\circ}$ peel-off were successfully implemented and gave repeatable results. Values measured with $90^{\circ}$ peel-off were significantly higher than those measured with $180^{\circ}$ peel-off $(0.085 \mathrm{~N} \pm 0.01 \mathrm{~N}$ and $0.065 \mathrm{~N} \pm 0.01 \mathrm{~N}$ respectively; $\mathrm{p}<0.05$ ) (Fig. 2B). The $90^{\circ}$ peel-off configuration was used for successive experiments due to easier handling during the test.

In order to determine whether this method would allow to quantify the integration derived from the matrix deposited at the osteochondral interface, we assessed both cell-free constructs and cell-based constructs cultured for 2 or 4 weeks in Chondrogenic medium (Fig. 2C). For this experiment, HAC from two different donors were used. Histological assessment of constructs confirmed the presence of cartilaginous matrix at the osteochondral interface, with 
the most intense staining for GAG in constructs cultured for 4 weeks in Chondrogenic medium (data not shown). The baseline peel-off force of cell-free constructs averaged $0.08 \mathrm{~N}$ \pm 0.01 and progressively increased for constructs generated with cell-seeded matrices over the two culture time points $(0.25 \mathrm{~N} \pm 0.01$ and $0.57 \mathrm{~N} \pm 0.11$ respectively). These results indicate that the test was capable to quantify the extent of integration between the two layers, upon matrix deposition at the interface (Fig. 2C).

\section{Modulation of the functionality of OC graft by preculturing the chondral layer}

\section{Macroscopic examination}

The technique to generate the OC grafts demonstrated to be easy to perform and gave reproducible results in terms of shape of the grafts. The cartilaginous layer was glossy and resilient when probed with a pair of forceps. Macroscopic observation revealed some areas of discontinuity at the cartilage/bony interface in samples of Group 3, probably due to a certain irregularity in the shape of the generated constructs that did not allow for precise shape matching with the upper surface of the bony scaffold. In contrast, OC samples of Group 1 and 2 appeared more regular (Fig. 3A).

\section{Histological and Immunohistochemical analyses}

Group 1 constructs contained a faintly Safranin-O stained extracellular matrix (ECM) mainly confined within the cartilage/bony interface. Group 2 constructs, instead, contained cartilaginous matrix intensely stained for GAG, comparable to that of chondral-only controls, which extended also to the cartilage/bony interface. Group 3 constructs displayed a faintly stained cartilaginous matrix, exclusively confined to the chondral layer, and including cells with picnotic-necrotic appearance (Fig. 3B).

Immunohistochemical analysis indicated that the ECM was stained for type II collagen only in the cartilage layers of Group 2 constructs, although less intensely then control samples (Fig. 3C). In contrast, type I collagen was negative in all the constructs (data not shown).

\section{Integration tests}


The peel-off test indicated that in Group 1 and Group 2 integration forces were significantly higher than in Group 3 constructs (up to 2.5- and 3.2-fold respectively for peak force and total energy of integration) (Fig. 4). Interestingly, peak force of Group 3 constructs was similar to that of cell-free constructs, suggesting that prolonged pre-culture did not result in a biological bonding between the two layers of the graft.

\section{Biochemical analysis}

DNA content of chondral layers from Group 2 and 3 were statistically significantly higher (3.8- and 2.2-fold respectively) that those from Group 1. Conversely, DNA contents in the bony layers of Group 1 were significantly higher than those of Group 2 and 3 (up to 2.1-fold) (Fig. 5A). These results suggest that in Group 1 constructs cells infiltrated more extensively the pores of the bony scaffold, while they remained more confined within the cartilage layers of the precultured constructs (Group 2 and 3). Consistent with the histological staining intensity and pattern, GAG amounts accumulated in the chondral layer of Group 2 constructs were remarkably and significantly higher than those measured in the chondral layers of Group 1 and 3 constructs (up to 5.9-fold), whereas GAG amounts accumulated in the bony layers of Group 1 constructs were significantly higher that those measured in the bony layers of Group 2 and 3 constructs (up to 2-fold) (Fig. 5A). The ratio of GAG/DNA, used as the quality index for the chondral layers [19] indicated that chondral tissues from the Group 2 constructs contained significantly higher contents of GAG as compared to those from Group 1 or 3 constructs (2.0- and 3.2-fold respectively) and approached levels measured in chondral-only control constructs (Fig. 5B).

\section{Gene expression}

Chondral and bony layers from Group 2 constructs were assessed via RT-PCR to define the stage of differentiation of cells located in the Chondro-Gide ${ }^{\circledR}$ matrices or in the Tutobone ${ }^{\circledR}$ blocks. The expression of type I and type X collagen in the cartilage layers was similar to that of bony layers and of control chondral constructs. In contrast, type II collagen and BSP 
expression were differentially regulated by the substrates used. In particular, type II collagen in the chondral layer was 73.1-fold higher than in the bony layer and, conversely, BSP in the bony layer was 11.1-fold higher than in the chondral layer (Fig. 6). These results suggest that HAC in the bony layers were less capable to re-differentiate into the chondrogenic lineage and appeared to acquire a more osteogenic phenotype.

\section{Effect of the bony scaffold on cartilaginous tissue development}

We have observed that the ECM deposited by HAC proximally to the trabeculae of the Tutobone $^{\circledR}$ was faintly stained for GAG and type II collagen, while that produced by HAC on Chondro-Gide ${ }^{\circledR}$ was more strongly stained for the same cartilaginous macromolecules (Fig. 3B,C). We thus performed an experiment aimed at assessing whether components released by Tutobone ${ }^{\circledR}$ may have caused the observed reduced HAC chondrogenesis. Cell-seeded matrices were cultured in the same wells together with Tutobone ${ }^{\circledR}$ blocks (co-culture Group) or alone (control Group) in Chondrogenic medium for 5 weeks (Fig. 1B). The resulting cartilaginous tissues were stained for GAG and type II collagen with comparable intensity (Fig. 7A, 7B). Biochemical analysis demonstrated that co-culture and control cartilaginous tissues contained similar amounts of DNA and GAG (Fig. 7C). These results suggest that the observed reduced chondrogenesis of HAC proximal to the bony layer is not caused by a release of chemicals from the Tutobone ${ }^{\circledR}$, but probably by a limited exchange of nutrients, caused by the presence of an adjacent material.

\section{DISCUSSION}

In this study we succeeded in engineering in vitro functional osteochondral (OC) composites, where the chondral layer was efficiently developed and biologically integrated with a bony substrate through the extracellular matrix produced by human articular chondrocytes (HAC). In particular, in our experimental setting, which was based on clinically compliant cells and biomaterials, we demonstrated that 3 days of separate pre-culture of the chondral layer prior 
to its fusion with the bony layer yielded a superior performance as compared to no pre-culture or 14 days pre-culture.

Since delamination of the engineered cartilage has been recognized as one of the most frequent complications of ACI [25], integration between the two layers of an OC construct is a key issue in engineering composite grafts $[5,9,26]$. The potential drawback of a strategy relying on the use of two separate and not monolithic scaffolds for the generation of OC constructs must be compensated by an efficient integration between the two layers. An innovative approach to the problem of tissue bonding has been reported by Wang et al. using the biopolymer chondroitin sulphate as a biochemical glue [27]. Instead, our cell-based strategy relies upon the temporary adhesion provided by fibrin glue and on the cartilaginous extracellular matrix synthesized by HAC, which is expected to be further consolidated over time following implantation.

In order to assess the integration strength between the chondral/bony layers, we developed a "peel-off test” which, unlike previously proposed methods [27-30], is compatible with a membranous structure of the chondral layer and provides quantitative data. In this context, it is relevant to underline that the minimum strength of integration needed at the time of implantation, which should support graft resistance to the shear forces acting in the joint and should determine the appropriate post-operative loading regime, has not yet been established [9]. On the other hand, the value we obtained at the end of the in vitro culture was up to 10fold that provided by acellular fibrin glue, which is currently used in clinical practice for the sealing of chondral grafts. Translated to a clinical scenario, this suggests that the developed OC grafts, thanks to the presence of newly formed extracellular matrix anchoring the two layers, could allow for implementation of less cautious post-operative rehabilitation protocols. The influence of certain natural and synthetic subchondral scaffolds on engineered cartilage development has been already reported [8,9]. Biphasic grafts made of various biomaterials and porcine chondrocytes have been evaluated by Wang et al. [8]. In that study the authors 
obtained better results with collagen-hydroxyapatite subchondral scaffolds in terms of chondral layer quality and macroscopic integration, as compared to those obtained with polymeric materials such as poly-L-lactide and poly-D,L-lactide. This motivated the choice for the scaffolds in our cell-based graft. The influence of a substrate based on devitalized bone on cartilaginous tissue development in OC constructs was recently addressed by Lima et al. in a different model (bovine chondrocytes from 1-3 weeks-old calves seeded in agarose gel) [9]. In their model they showed a lower quality of the cartilaginous layer of OC grafts including that bony substrate, but also in chondral samples co-cultured with their bony substrate. To improve these results they proposed to use porous tantalum metal instead of devitalized bone. In our OC model, the presence of Tutobone ${ }^{\circledR}$ did not reduce the GAG accumulation of the chondral layer, if that was pre-cultured for 3 days. Based on the co-culture experiment (Fig. 1B), the observed slight reduction in the staining for type II collagen should not be attributed to the presence of the Tutobone ${ }^{\circledR}$ material per se, but is likely a result of reduced mass transfer due to the presence of an adjacent material, and prompts for future studies possibly introducing perfusion-based bioreactor systems to overcome this limit [31].

Our proposed method for producing osteochondral constructs also has certain limitations: the thickness of the chondral layer of the graft (around $1 \mathrm{~mm}$ ) makes it suitable only for the regeneration of cartilage defects in small joints (e.g., ankle) and in none of our groups did we manage to duplicate the morphology of the tidemark, even if an upregulation of the BSP gene was noticed in the chondrocytes in contact with the bony scaffold. However, an engineered OC graft can further develop once implanted in the joint and undergo a remodelling process that can even increase tissue thickness and recreate the bone-cartilage interface, as shown by Kandel et al [7]. This process could be facilitated by local mechanical loading in the 'in vivo bioreactor' of the joint, and further emphasizes the need to generate grafts that could tolerate joint loading as early as possible after implantation. 


\section{CONCLUSIONS}

The OC grafts engineered according to the described method display several features advocating a large potential for clinical implementation, including: (i) a biological bonding of the chondral layer with the bony scaffold through the extracellular matrix produced by human cells; (ii) a clinically relevant diameter; (iii) a suitable stability allowing easy surgical handling and the possibility of insertion into the joint through a recently developed technique [32]; and (iv) a versatility in adaptation to different joints, thanks to the use of bony layers with appropriate curvatures, quantitatively defined using anatomical data [33]. These results suggest that this model, which is based on human cells and biomaterials currently in clinical use, could be relatively early introduced in clinical practice and represents a step ahead towards the more ambitious engineering of biological substitutes for total joint replacement.

\section{ACKNOWLEDGMENTS}

The study was partially financed by the Swiss National Science Foundation (Grant No. 3200B0-110054), by the "Deutsche Arthrose-Hilfe e.V.” and by the Hardy \& Otto Frey-Zünd Foundation. We gratefully thank Baxter Healthcare for kindly providing Tisseel ${ }^{\circledR}$ fibrin glue; Geistlich Pharma AG for the generous supply of Chondro-Gide ${ }^{\circledR}$ matrices; Tutogen Medical GmbH and Novomedics GmbH for the generous supply of Tutobone ${ }^{\circledR}$ scaffolds. 